\begin{abstract}
Submitted: 11.04.2018

Accepted: 15.07.2018

Published: 31.12.2018

Keywords ultrasound, scaphoid fracture, diagnosis, accuracy

\section{The role of ultrasonography in the diagnosis of occult scaphoid fractures}

\author{
Mohammed Ali ${ }^{1}$, Mujtaba Ali ${ }^{1}$, Ahmed Mohamed², Syed Mannan, \\ Farshid Fallahi ${ }^{1}$ \\ ${ }^{1}$ North Cumbria University Hospital NHS Trust, Carlisle, United Kingdom
${ }^{2}$ Health Education North East, Newcastle upon Tyne, United Kingdom \\ Correspondence: Mohammed Ali, Trauma and Orthopaedics, North Cumbria University \\ Hospitals NHS Trust, Newtown Road, Carlisle, UK, CA2 7HY; \\ e-mail:mohammedkhider84@hotmail.com,mohammedkhider84@hotmail.com
}

DOI: $10.15557 /$ JoU.2018.0047

Abstract

Background: Ultrasonography is widely utilized by emergency physicians and radiologists to diagnose various orthopaedic diseases, including fractures. We aim to derive a definitive estimate of the diagnostic accuracy of ultrasonography in clinically suspected scaphoid fractures. Methods: We undertook a systematic review and meta-analysis of included diagnostic cohort studies that discussed the use of ultrasonography in the diagnosis of occult scaphoid fractures. We searched the National Institute for Health and Care Excellence database using the Healthcare Databases Advanced Search tool. In addition, we utilized the PubMed database to search the Medical Literature Analysis and Retrieval System Online, Excerpta Medica database, Cumulative Index of Nursing and Allied Health and Allied and Complimentary Medicine databases. Studies were included if they discuss the role of ultrasound imaging in the diagnosis of scaphoid fractures based on cortical interruption, radio-carpal effusion and scapho-trapezium-trapezoid effusion. Quality assessment was performed using the methodological index for non-randomized studies scoring system. Results: 6 non-randomized control studies met the inclusion criteria. Collectively, these included 236 patients with a mean age ranging from 18 to 41.2 years. The quality of these articles ranged between moderate and high based on the methodological index for nonrandomized studies score. The mean sensitivity was $88.95 \%$ (standard deviation 10.03) and mean specificity was $89.50 \%$ (standard deviation 12.21). Conclusion: The current literature reveals high sensitivity and specificity in the use of ultrasonography in scaphoid fracture diagnosis. However, multiple factors including technical differences in ultrasound machines and probes, small sample sizes and variability of subsequent confirmatory tests have created a challenge in determining the ultimate reliability of ultrasonography in the diagnosis of occult scaphoid fractures. Considering these factors and limitations, large-sample and high-quality clinical trials are needed to adequately assess its reliability for this purpose. One stop clinics, in the authors' opinion, would be an ideal setting for its introduction as well as for future trials.
\end{abstract}

\section{Introduction}

The scaphoid bone plays a pivotal role in the wrist carpal complex as it preserves the normal alignment of the carpus creating normal wrist motion ${ }^{(1)}$. Early diagnosis of a scaphoid fracture is critical to promoting healing and restoring alignment and hence conducive to a good clinical outcome. In the case of a missed diag- nosis, inter-fragmentary motion will affect the fracture healing process and could lead to complications such as avascular necrosis, mal-union, delayed union and nonunion. Furthermore, kinematic abnormalities resulting from malalignment could result in instability of the wrist carpal complex. Complications resulting from a missed diagnosis of a scaphoid fracture can lead to significant long-term disability ${ }^{(2-4)}$. Early diagnosis and treatment 
improves clinical outcome and reduces complication rates. Imaging plays a vital role in diagnosis as it can be difficult to assess patients clinically with relatively innocuous symptoms.

Some authors have argued that subtle fractures which are not visible on the initial radiographs are likely to heal spontaneously without immobilisation; but this view is not widely shared as the scaphoid bone is affected by nearly every motion of the hand, wrist and forearm, subsequently causing movement and pressure on the fracture line ${ }^{(5)}$. Routinely, scaphoid views would be ordered as a first line investigation and in the majority of cases these fractures can be diagnosed primarily if radiographs are of good quality. When radiographs are inconclusive and the patient is symptomatic a second line of imaging investigation should be considered. Bone scintigraphy (BS), computed tomography (CT) and magnetic resonance imaging (MRI) can be helpful when repeat radiographs are not diagnostic $^{(6)}$. Some authors have advocated the role of ultrasonography in diagnosing fractures based on many factors. Cortical bone can be reliably visualized on ultrasound (US) given its reflective acoustic characteristics; this renders ultrasound imaging of cortical bone a highly specific means of identifying fractures as small as $1 \mathrm{~mm}^{(7)}$. Currently, ultrasound is widely utilized by emergency physicians to aid diagnosis of a variety of clinical entities with a high degree of reliability. The constant advancement of ultrasound imaging and its availability have led to its increased utilisation as a first line diagnostic modality amongst clinicians in general. This has subsequently led to more appropriate use of this diagnostic modality. Furthermore, ultrasound is non-invasive, radiation free and relatively inexpensive. On the other hand, ultrasound has limited penetration abilities and areas of deep bone or thick layers of soft tissues may not be well visualized ${ }^{(8)}$. Ultrasound is also highly operator-dependent compared to other imaging modalities.

The purpose of this review is to systematically evaluate the literature concerning the role of ultrasound in the diagnosis of scaphoid fractures.

\section{Methods}

We searched the National Institute for Health and Care Excellence (NICE) database using the Healthcare Databases
Advanced Search (HDAS) tool. In addition, we utilized the PubMed database to search the Medical Literature Analysis and Retrieval System Online (MEDLINE), Excerpta Medica database (EMBASE), Cumulative Index of Nursing and Allied Health (CINAHL) and Allied and Complimentary Medicine (AMED) databases.

The search of these databases was conducted from their year of inception to February 2018. Broad search keywords were used, rather than specific terms, to ensure no articles were missed. Abstracts of all studies identified by the search strategy were examined and, if relevant, the papers were then read in full. The reference lists of these papers were also searched. Study selection, assessment of quality, and data extraction were performed independently by two authors: Mohammed Ali (MA) and Mujtaba Ali (Muj A). The Preferred Reporting Items for Systematic Review and Meta-Analysis (PRISMA) methodology guidance was employed $^{(9)}$. Studies were included if they discuss the use of ultrasound to diagnose scaphoid fractures based on cortical interruption (CI), radio-carpal effusion (RCE) and scapho-trapezium-trapezoid effusion (STTE). We excluded single case reports, reviews, conference abstracts, studies on cadavers, technical notes and articles not published in English. Studies utilising tenderness induced by direct pressure with the ultrasound probe were also excluded.

\section{Data extraction and synthesis}

One author (MA) extracted data using a standardized form for each of the following: author, year of publication, study design, sample size, demographics, operator specialty, confirmation test, sensitivity and specificity of reported values. This was verified by a second author (Muj A).

\section{Quality assessment}

As all the included articles are non-randomized control trial studies, the quality assessment was performed using the methodological index for non-randomized studies (MINORS) score ${ }^{(10)}$. It has 12 domains for which noncomparative studies use the first 8 domains. Each domain is scored out of 2 with ideal scores being at least 16 for non-comparative studies and 24 for comparative studies. The investigators discussed scores where more than

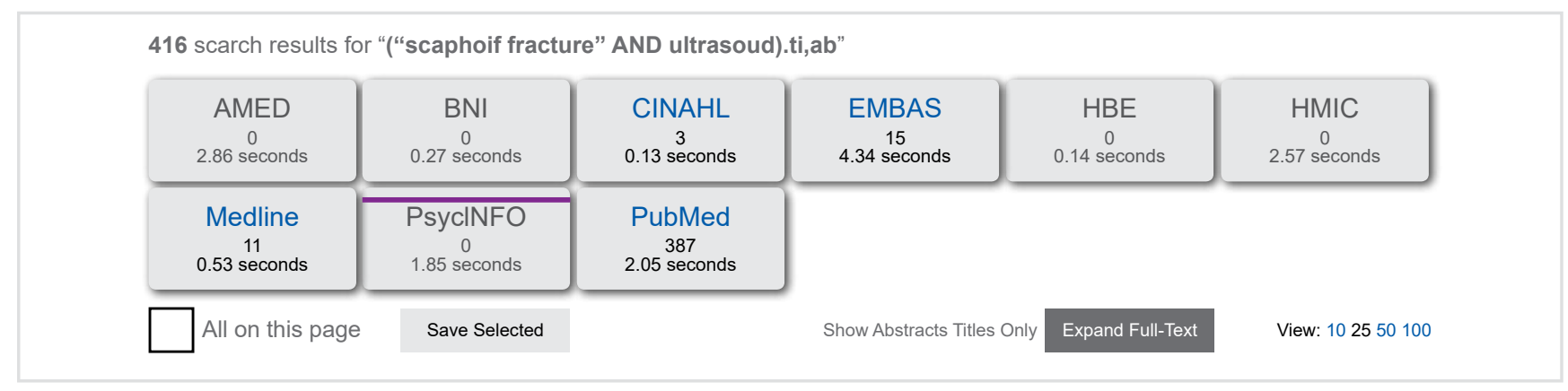

Fig. 1. Search results 
two-point difference was recorded, until an agreement has been reached.

Systematic review registration:

PROSPERO CRD42017082758.

\section{Results}

\section{Search results}

The queries used in the NICE HDAS database were "scaphoid fracture" and "ultrasound scan"; these resulted in 416 articles. The search results are outlined in Figure 1.

Duplicates, non-clinical and studies unrelated to US were excluded. In addition, we excluded reviews, operative technique articles, case reports and ultrasound studies not utilising CI, RCE and STTE (Fig. 2). 6 articles ${ }^{(11-16)}$ met the inclusion criteria for this review.

\section{Summary of studies}

In total, 6 non-RCT studies were included for analysis ${ }^{(1-16)}$. These articles included 236 patients, with sample sizes ranging from 15 to 63 patients per study and mean age ranging from 18 to 41.2 years. The quality of these articles ranged between moderate and high based on the MINORS score (Tab. 1).

\section{Symptoms and inclusion criteria}

All authors agreed on tenderness and swelling of the anatomical snuff box alongside tenderness on axial loading as criteria for clinical inclusion ${ }^{(11-16)}$. Some authors added restriction of wrist movement ${ }^{(11,15)}$ and wrist pain ${ }^{(15,16)}$. All these studies focused on detecting cortical interruptions and joint effusions on ultrasonography.

\section{Confirmation test/ control}

Herneth $^{(11)}$ and Yildirim ${ }^{(16)}$ used MRI to confirm the ultrasound findings, Fusetti ${ }^{(14)}$ and Platon ${ }^{(15)}$ CT scan, Senall ${ }^{(13)}$ repeated the plain films while in Hauger' ${ }^{(12)}$ study there was no uniformed confirmatory test (Tab. 2).

\section{Sensitivity and specificity}

Herneth and Senall used the same ultrasound machine with similar low frequency probes $(5 \mathrm{MHz})$ and both had

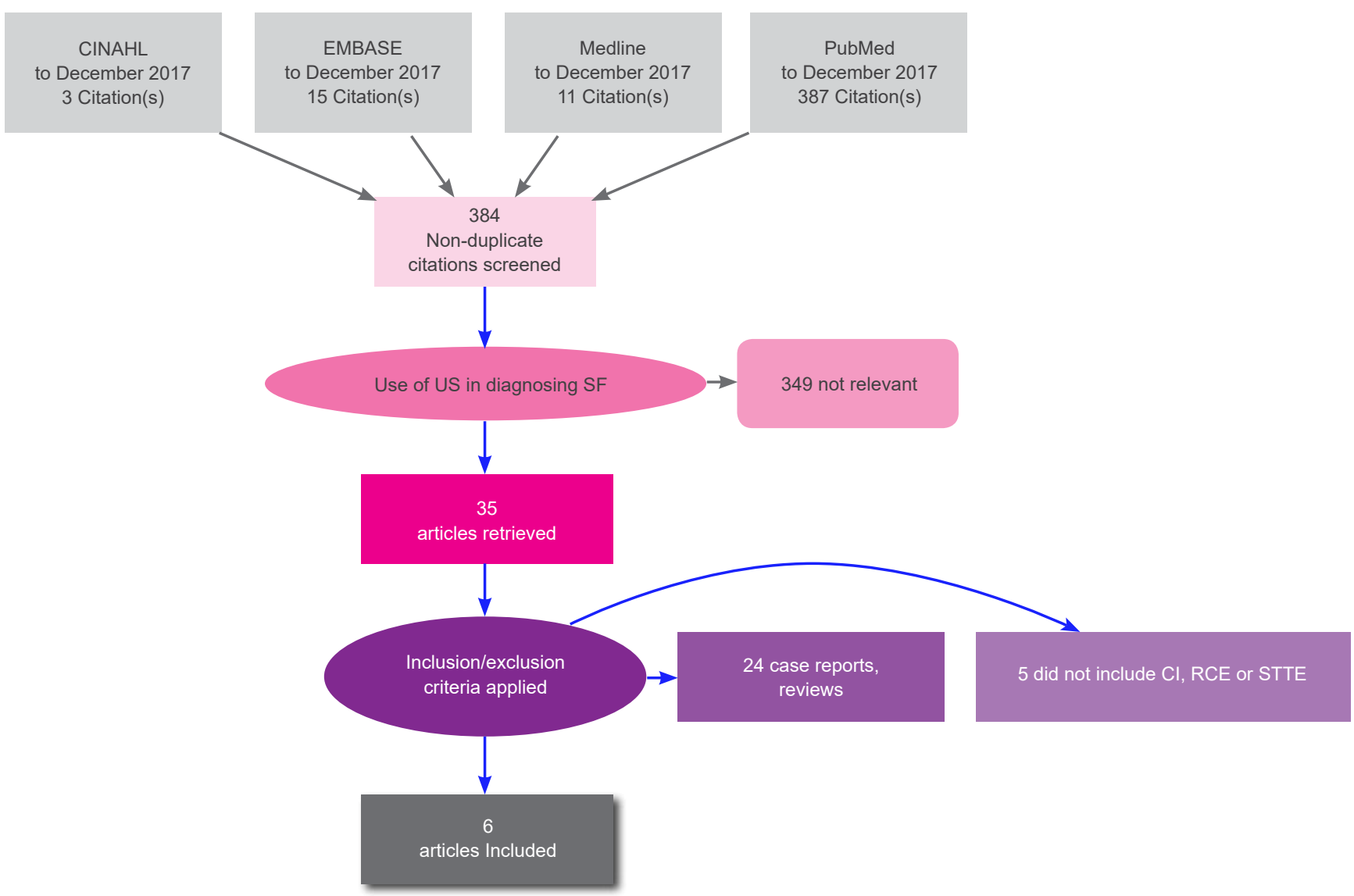

Fig. 2. Study selection process 


\begin{tabular}{|l|c|c|c|c|c|}
\hline Author, year & RCT/CS & Number of patients & Mean age & Male/female & Minors \\
\hline Herneth et al. $\mathbf{2 0 0 1}^{(11)}$ & CS & 15 & 23.5 & $7 / 8$ & $35 / 19$ \\
\hline Hauger et al. $\mathbf{2 0 0}^{\mathbf{2 ( 1 2 )}}$ & CS & 54 & 35 & not available \\
\hline Senall et al. $\mathbf{2 0 0 3}^{(13)}$ & CS & 18 & 42 & $13 / 11$ & 10 \\
\hline Fusetti et al. $\mathbf{2 0 0 5}^{(14)}$ & CS & 24 & 41,2 & $29 / 33$ & 14 \\
\hline Platon et al. $\mathbf{2 0 1 1}^{(15)}$ & CS & 62 & $>18$ & not available \\
\hline Yildirim et al. $\mathbf{2 0 1 3}^{(16)}$ & CS & 63 & 12 \\
\hline
\end{tabular}

Tab. 1. Description of included articles

\begin{tabular}{|c|c|c|c|c|c|}
\hline Author, year & Baseline test & Exam included & Waiting time & US device & Confirmation test \\
\hline Herneth et al. 2001(11) & scaph views & $\mathrm{Cl}, \mathrm{SPE}$ & 72 hours & ATL, HDI $3000,10-5 \mathrm{MHz}$ & MRI \\
\hline Hauger et al. $2002^{(12)}$ & scaph views & $\mathrm{Cl}, \mathrm{SPE}$ & within 7 days & HDI $3000 \& 5000,12 \mathrm{MHz}$ & XR, CT, MR, B-scan \\
\hline Senall et al. $2003^{(13)}$ & scaph views & $\mathrm{Cl}, \mathrm{SPE}$ & 4.7 days & ATL, HDI $3000,10-5 \mathrm{MHz}$ & $\mathrm{XR}$ \\
\hline Fusetti et al. $2005^{(14)}$ & scaph views & $\mathrm{Cl}, \mathrm{RCE}, \mathrm{STTE}$ & not mentioned & $\begin{array}{l}\text { ATL-Philips 5000, } \\
\quad 7-15 \mathrm{MHz}\end{array}$ & CT \\
\hline Platon et al. $2011^{(15)}$ & scaph views & $\mathrm{Cl}, \mathrm{RCE}, \mathrm{STTE}$ & 3 days & $\begin{array}{c}\text { Prosound SSD-5000, } \\
5-13 \mathrm{MHz}\end{array}$ & $\mathrm{CT}$ \\
\hline Yıldırım et al. $2013^{(16)}$ & $\begin{array}{l}\text { Clinical } \\
\text { exam }\end{array}$ & $\mathrm{Cl}, \mathrm{RCE}, \mathrm{STTE}$ & 24 hours & DC3, Mindray, 5-13 MHz & MRI \\
\hline
\end{tabular}

Tab. 2. Ultrasound characteristics and confirmatory tests

the same sensitivity (78\%) which was the lowest amongst the other studies. Fusetti and Hauger used high frequency probes (15 MHz and $12 \mathrm{MHz}$ respectively) and they both achieved $100 \%$ sensitivity (Tab. 3 and Tab. 4) (Fig. 3).

\section{Discussion}

The use of bedside musculoskeletal ultrasound has been supported by many authors based on the availability and practicality of this technique. Backhaus et al. states that the hyperechoic reflection of bony cortex in ultrasound images yields valuable diagnostic information ${ }^{(17)}$. On ultrasound, fracture can manifest itself as disruption or irregularity amongst other bone pathologies such as exostoses and osteophytes. In addition, some bone tumors have also been examined with US; this was found to be useful as it allows assessing blood flow to the tumor using Doppler imaging ${ }^{(18)}$. Sinha et al. ${ }^{(19)}$ described it as a legitimate tool for fracture diagnosis that can be used instead of conventional radiography on certain occasions. They advocated the use of bedside ultrasound for extremity trauma in acute settings as it has several advantages. These include a shorter waiting time for diagnosis, especially in busy facilities or when there is no access to radiography. Furthermore, it helps avoiding unnecessary exposure to radiation, particularly in the paediatric population. This review has identified a small number of non-RCTs ${ }^{(11-16)}$ reporting the use of ultrasound in the diagnosis of scaphoid fractures. Below we highlight a number of interesting observations from the articles reviewed.

In 2001, Herneth et al. ${ }^{(11)}$ evaluated the diagnostic accuracy of high-spatial-resolution sonography (HSR-S) in the diagnosis of scaphoid fractures. 15 symptomatic patients with inconclusive radiographs were examined using US imaging by two blinded experienced radiologists. A fracture was deemed present when HSR-S demonstrated cortical discontinuity and/or periosteal elevation. Subsequently, MRI confirmed 9 of 15 patients to have scaphoid fractures. $7(78 \%)$ of these 9 patients had positive findings on HSR-S. Furthermore, 2 (50\%) of 4 radiographically occult scaphoid fractures were illustrated with HSR-S. Despite the small number of patients, Herneth concluded that HSR$\mathrm{S}$ is a trustworthy examination and should be performed before considering a CT or MRI scan. Another study by Hauger et al. in $2002^{(12)}$, concluded that HSR-S is a reliable and accurate method of evaluating occult fractures of the scaphoid waist. They could diagnose occult fractures at an early stage and they considered cortical disruption 
as the key to diagnosis. They did not take soft tissue abnormalities alone as sufficient evidence as these can be secondary to other internal injuries such as ligamentous injuries. Senall et al. in $2003^{(13)}$ evaluated 18 symptomatic wrists using physical examination, conventional radiography and HSR-S. Ultrasound was able to identify 7 of 9 cases that were eventually positive for scaphoid fracture on plain radiographs. Ultrasound was read correctly as no fracture in 8 of $9 x$-ray-negative cases. The 1 false-positive case had radio-scaphoid arthrosis and radial wrist swelling. A major limitation of this study was that the final diagnosis was entirely based on plain radiographs. Fusseti et al. in $2005^{(14)}$ conducted a study to evaluate the diagnostic accuracy of HSR-S in subtle scaphoid fractures. They classified patients with subtle scaphoid fractures into three levels of suspicion (high, intermediate and low) based on the US findings. These included CI, RCE and STTE. The three aforementioned criteria were interpreted as being highly indicative of a scaphoid fracture. A high sonographic index of suspicion was correlated with $100 \%$ sensitivity, specificity, positive predictive value, and negative predictive value. When they compared the results with CT scan findings, they concluded that HSR-S has $100 \%$ sensitivity and $79 \%$ specificity. Fusetti et al. ${ }^{(14)}$ stated that there were several limitations to this examination. These included the impossibility to screen the overall contour of the bone due to movement restriction secondary to pain. This was particularly evident with ulnar deviation of the wrist which is a very important position when evaluating the most proximal part of the scaphoid bone. In addition, they listed the scaphoid tubercle and the scapho-lunate

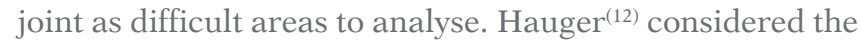
scaphoid tubercle area as a difficult area to examine due to the natural irregularity of the scaphoid at this site which may mimic cortical disruption. Furthermore, it is difficult to demonstrate the extent and configuration of the fracture and, at the end of the day, it is a highly operator-dependant procedure necessitating adequate training and experience amongst the performing clinicians.

Platon et al. in 2011(15) evaluated 62 consecutive patients with suspected scaphoid fractures using ultrasound prior to $\mathrm{CT}$ scan. The radiologist looked for cortical interruption along with a radio-carpal or scapho-trapezium-trapezoid effusion.

Fractures were put into two groups according to their potential for complication: high (proximal or waist) and low (distal or tubercle). CT scans showed scaphoid fracture in $13(21 \%)$ patients: 8 high potential (3 proximal pole and 5 waist) and 5 low potential ( 3 distal pole and 2 at the tubercle). US was $92 \%$ sensitive (12/13) in demonstrating a scaphoid fracture overall. It was $100 \%$ sensitive $(8 / 8)$ in demonstrating a fracture with a high potential for complication. Platon considered these results to be related to the anatomical position of the scaphoid. As the scaphoid waist was the easiest to view on US, signs of fracture were reliably identified at this site. On the other hand, scanning the distal pole or the tubercle was more technically challenging. In a recent study, Yildirim ${ }^{(16)}$ evaluated 63 patients with suspected scaphoid fractures using bedside ultrasonography and MRI. A scaphoid fracture was diag-

\begin{tabular}{|c|c|c|c|c|c|}
\hline Author, year & US operator & Sensitivity & Specificity & $\begin{array}{c}\text { Positive predictive } \\
\text { value }\end{array}$ & $\begin{array}{c}\text { Negative predictive } \\
\text { value }\end{array}$ \\
\hline Herneth $2001^{(11)}$ & Rad & $78 \%$ & $100 \%$ & $100 \%$ & $75 \%$ \\
\hline Hauger $2002^{(12)}$ & MSK Rad & $100 \%$ & $98 \%$ & $83 \%$ & $100 \%$ \\
\hline Senall i wsp. $2003^{(13)}$ & MSK Rad & $78 \%$ & $89 \%$ & $88 \%$ & $80 \%$ \\
\hline Fusetti $2005^{(14)}$ & MSK Rad & $100 \%$ & $79 \%$ & $56 \%$ & $100 \%$ \\
\hline Platon et al. $2011^{(15)}$ & Rad & $92 \%$ & $71 \%$ & $46 \%$ & $97 \%$ \\
\hline Yıldırım et al. $2013^{(16)}$ & AE Doctor & $85,70 \%$ & $100 \%$ & $100 \%$ & $100 \%$ \\
\hline
\end{tabular}

Tab. 3. Sensitivity and specificity

\begin{tabular}{|l|c|c|c|c|c|}
\hline & $\boldsymbol{N}$ & Minimum & Maximum & Mean & Std. deviation \\
\hline Sensitivity & 6 & $78.00 \%$ & $100.00 \%$ & $88.9500 \%$ & $10.03868 \%$ \\
\hline Specificity & 6 & $71.00 \%$ & $100.00 \%$ & $89.5000 \%$ & $12.21065 \%$ \\
\hline Positive predictive value & 6 & $46.00 \%$ & $100.00 \%$ & $78.8333 \%$ & $22.78962 \%$ \\
\hline Negative predictive value & 6 & $75.00 \%$ & $100.00 \%$ & $92.0000 \%$ & $11.40175 \%$ \\
\hline
\end{tabular}

Tab. 4. Descriptive statistics 


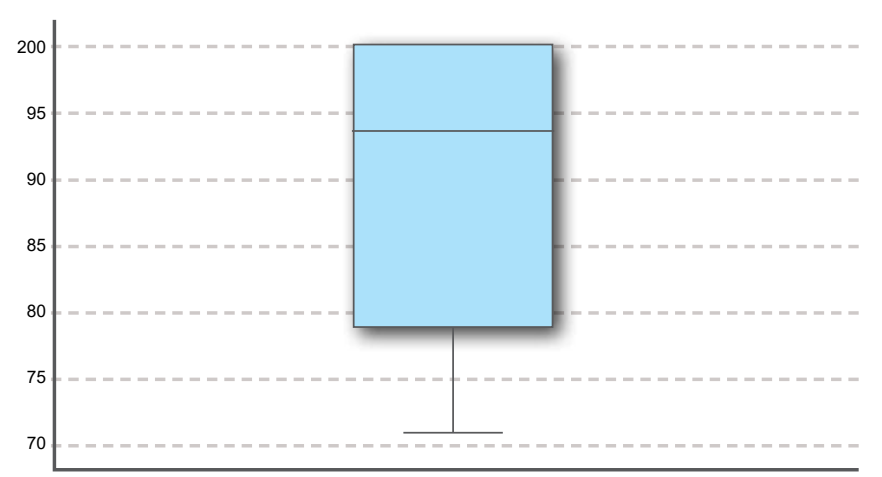

Specificity

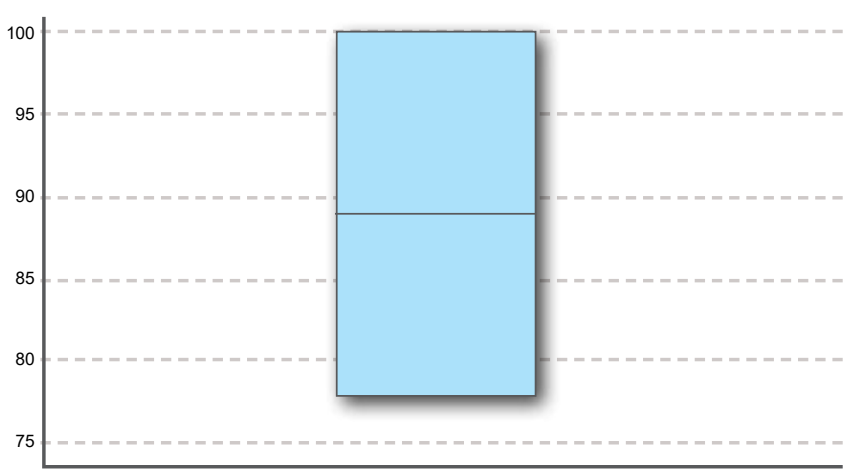

Sensitivity

Fig. 3. Descriptive statistics

nosed by MRI in 14 patients. Ultrasound revealed signs indicative of scaphoid fracture in 12 of the 14 patients. Of these, 12 were corroborated by MRI and none found to be negative. In 45 cases, ultrasound did not reveal scaphoid fracture, of which two were deemed to have a scaphoid fracture following the MRI scan. The limitation of this study was a lack of blinding in the examining emergency physicians.

The stage at which ultrasonography can be incorporated into the diagnostic process of suspected scaphoid fracture is a matter for debate. Current practice in the United Kingdom encountered by the authors in multiple centres entails initial conventional radiography in the acute setting followed by repeat radiographs after a set interval if the patient continues to be symptomatic. If the second set of radiographs remains inconclusive, and after confirming suspicion by orthopaedic surgeons, a more superior imaging modality is considered. Commonly this would involve MRI although CT is also widely utilized for this purpose. During this period, which can often be lengthy, the affected wrist and hand will commonly be immobilized. Ultrasonography can potentially be introduced at different stages within this pathway. The first consideration is for ultrasonography to be utilized as a primary imaging investigation by emergency care physicians. The superiority of ultrasonography over conventional radiography concluded in this review would be the main advantage of this approach as it can potentially facilitate either earlier confirmation or exclusion of the diagnosis and hence either facilitate earlier management or avoid prolonged periods of immobilisation respectively. The main limitation of this would be the need for adequate training required for emergency physicians, particularly that ultrasonography is highly user-dependent. The second consideration would be the introduction of ultrasonography as a secondary confirmatory test after failure of conventional radiography. The main advantage to the second consideration would be reducing the number of costly investigations such as MRI while also reducing waiting times as ultrasonography is more accessible. Limitations to the second consideration would be a lack of large volume clinical trials that support the reliability of ultrasonography for this purpose. An increasing practice in recent years has been the introduction of one stop clin- ics run collaboratively by orthopaedic surgeons and with musculoskeletal radiologists available in the clinic. The incorporation of ultrasonography in the setting of a one stop clinic would be our third consideration. In the authors' opinion, this would be the most ideal setting for the introduction of ultrasonography into the diagnostic process of suspected scaphoid fractures given the instant availability of an experienced musculoskeletal radiologist to perform the examination. Furthermore, a combined decision can be made at the time as to the necessity of further imaging modalities such as MRI. This would also be an ideal setting to perform large volume prospective clinical trials.

The choice of secondary confirmatory test is an important consideration in future clinical trials looking into ultrasonography in the diagnosis of radiographically occult scaphoid fractures. The confirmatory test varied in the studies reviewed in this article. Bone scintigraphy, $\mathrm{CT}$ and MRI have been widely used and studied for this purpose. Nonetheless, uncertainty remains regarding the most appropriate ${ }^{(20)}$. In addition to sensitivity and specificity, other factors should be taken into account when deciding upon the confirmatory imaging test. These include invasiveness and exposure to ionising radiation. Clinicians should be aware of advantages and limitations of each modality. It is the authors' view that MRI would constitute a suitable confirmatory test in future studies given its superior sensitivity and lack of ionising radiation exposure.

\section{Conclusion}

Overall, not many authors have studied the role of ultrasound in the diagnosis of scaphoid fractures. The current literature has revealed that ultrasound has high sensitivity and specificity. Multiple factors including technical differences in ultrasound machines and probes, small sample sizes and variability of subsequent confirmatory tests have created a challenge in determining the ultimate reliability of ultrasonography in the diagnosis of occult scaphoid fractures. Considering these factors and limitations, largesample and high-quality clinical trials are needed to adequately assess its reliability for this purpose. The stage at which ultrasonography can be incorporated into the diag- 
nostic process of suspected scaphoid fractures is a debatable matter and would largely depend on local procedures and facilities available. Concluding an approach that could be generalized would be difficult and therefore, a selective approach by individual centres would seem appropriate at present. In the authors' opinion, combined one stop clinics would constitute the ideal setting for the introduction of ultrasound diagnosis of scaphoid fractures if these are

\section{Reference}

1. Berdia S, Wolfe SW: Effects of scaphoid fractures on the biomechanics of the wrist. J Bone Joint Surg Br 2008; 90: 1205-1209.

2. Smith DK, Cooney WP, An KN: The effects of simulated unstable scaphoid fractures on carpal motion. J Hand Surg Am 1989; 14: 283-291.

3. Kozin SH: Incidence, mechanism, and natural history of scaphoid fractures. Hand Clin 2001; 17: 515-524.

4. Amadio PC, Berquist TH, Smith DK, Ilstrup DM, Cooney WP 3rd, Linscheid RL: Scaphoid malunion. J Hand Surg Am 1989; 14: 679-687.

5. Kaneshiro SA, Failla JM, Tashman S: Scaphoid fracture displacement with forearm rotation in a short-arm thumb spica cast. J Hand Surg Am 1999; 24: 984-991.

6. Rhemrev SJ, Ootes D, Beeres FJ, Meylaerts SA, Schipper IB: Current methods of diagnosis and treatment of scaphoid fractures. Int J Emerg Med 2011; 4: 4 .

7. Fontes-Pereira A, Matusin DP, Rosa P, Schanaider A, von Krüger MA, Pereira WCA: Ultrasound method applied to characterize healthy femoral diaphysis of Wistar rats in vivo. Braz J Med Biol Res 2014; 47: 403-410.

8. Patil P, Dasgupta B: Role of diagnostic ultrasound in the assessment of musculoskeletal diseases. Ther Adv Musculoskelet Dis 2012; 4: 341355.

9. Moher D, Shamseer L, Clarke M, Ghersi D, Liberati A, Petticrew M et al.: Preferred reporting items for systematic review and meta-analysis protocols (PRISMA-P) 2015 statement. Syst Rev 2015; 4: 1.

10. Slim K, Nini E, Forestier D, Kwiatkowski F, Panis Y, Chipponi J: Methodological index for non-randomized studies (minors): development and validation of a new instrument. ANZ J Surg 2003; 73: 712-716.

11. Herneth AM, Siegmeth A, BaderTR, Ba-Ssalamah A, Lechner G, MetzVM et al.: Scaphoid fractures: evaluation with high-spatial-resolution US initial results. Radiology 2001; 220: 231-235. locally available. Furthermore, this would be an ideal setting to perform large volume prospective unbiased clinical trials.

\section{Conflict of interest}

The authors declare that they have no conflict of interest.
12. Hauger O, Bonnefoy O, Moinard M, Bersani D, Diard F: Occult fractures of the waist of the scaphoid: early diagnosis by high-spatial-resolution sonography. AJR Am J Roentgenol 2002; 178: 1239-1245.

13. Senall JA, Failla JM, Bouffard JA, van Holsbeeck M: Ultrasound for the early diagnosis of clinically suspected scaphoid fracture. J Hand Surg Am 2004; 29: 400-405.

14. Fusetti C, Poletti PA, Pradel PH, Garavaglia G, Platon A, Della Santa DR et al.: Diagnosis of occult scaphoid fracture with high-spatial-resolution sonography: a prospective blind study. J Trauma 2005; 59 : 677-681.

15. Platon A, Poletti PA, Van Aaken J, Fusetti C, Della Santa D, Beaulieu JY et al:: Occult fractures of the scaphoid: the role of ultrasonography in the emergency department. Skeletal Radiol 2011; 40: 869-675.

16. Yıldırım A, Unlüer EE, Vandenberk N, Karagöz A: The role of bedside ultrasonography for occult scaphoid fractures in the emergency department. Ulus Travma Acil Cerrahi Derg 2013; 19: 241-245.

17. Backhaus M, Burmester G, Gerber T, Grassi W, Machold K, Swen Wet al.: Guidelines for musculoskeletal ultrasound in rheumatology. Ann Rheum Dis 2001; 60: 641-649.

18. Blankstein A: Ultrasound in the diagnosis of clinical orthopedics: The orthopedic stethoscope. World J Orthop 2011; 2: 13-24.

19. Sinha, TP, Bhoi S, Kumar S, Ramchandani R, Goswami A, Kurrey Let al.: Diagnostic accuracy of bedside emergency ultrasound screening for fractures in pediatric trauma patients. J Emerg Trauma Shock 2001; 4: 443-445.

20. Mallee WH, Wang J, Poolman RW, Kloen P, Maas M, de Vet HC et al:: Computed tomography versus magnetic resonance imaging versus bone scintigraphy for clinically suspected scaphoid fractures in patients with negative plain radiographs. Cochrane Database Syst Rev 2015; 6. DOI: 10.1002/14651858.CD010023.pub2 